\title{
ETNOVETERINÁRIA: A FITOTERAPIA NA VISÃO DO FUTURO PROFISSIONAL VETERINÁRIO
}

\author{
Katyane de Sousa Almeida \\ Pesquisadora da Universidade Federal do Amazonas-UFAM \\ katyanefreitas@gmail.com \\ Fagner Luiz da Costa Freitas \\ Professor da Universidade Federal do Amazonas-UFAM \\ fagner@ufam.edu.br
}

Tadeu Fladiner Costa Pereira

Departamento de Ciência Vegetais - UFERSA - Universidade Federal Rural do Semi-Árido fladner@gmail.com

\begin{abstract}
RESUMO - A Fitoterapia é um ramo da Etnoveterinária que trata das patologias que acometem os animais de forma natural e sem muitos gastos; como os futuros veterinários são os estudantes de hoje, se faz necessário que estes conheçam esta forma de terapia. Portanto, este trabalho objetivou recuperar e documentar os conhecimentos sobre a Etnoveterinária, assim como a importância desta no conjunto da sustentabilidade atual, avaliando o nível de conhecimento atual sobre fitoterapia e o seu uso pelos estudantes de veterinária da ESAM. O instrumento trabalhado foi a pesquisa direta através da aplicação de questionários ao corpo discente da veterinária da ESAM, durante o período de março a agosto de 2000. De acordo com os resultados, observou-se que o termo etnoveterinária não está amplamente difundido entre os estudantes de veterinária da ESAM, porém, a fitoterapia é de conhecimento de muitos e já utilizada por alguns, que citaram 24 plantas para o tratamento de diversas patologias animal, onde todas obtiveram sucesso no uso. A forma de divulgação mais indicada por eles foi a comunicação oral. Sobre a credibilidade da fitoterapia, 87,0 \% passariam a utilizar esta forma de tratamento após confirmação científica, explicando as vantagens e desvantagens desta aplicação. Pôde-se observar que a fitoterapia é conhecida entre o corpo discente de veterinária da ESAM, porém há necessidade da realização de novas pesquisas nesta área.
\end{abstract}

Palavras-chave: Etnoveterinária, Fitoterapia, Estudantes.

\section{Etnoveterynary: the phytotherapy in view of future veterinarian professional}

ABSTRACT - Phytotherapy is a branch of Ethnoveterinary that is about the pathologies that attack the animals in a natural way and without many expenses; as the future veterinarians are the students today, he makes himself necessary that these know this therapy form therefore, this work objectified to recover and to document the knowledge on Ethnoveterinary, as well as the importance of this in the group of the current sustentabilidade, evaluating the level of current knowledge on Phytotherapy and its use for the students of veterinary of ESAM. The worked instrument went to direct research through the application of questionnaires to the body student of the veterinary of ESAM, during the period of March to August of 2000. In agreement with the results, was observed that the term Ethnoveterinary is not thoroughly diffused among the students of veterinary of ESAM, even so, the Phytotherapy is of knowledge of many and already used by some, that mentioned 24 plants for the treatment of several pathologies animal, where everybody obtained success in the use. The popularization form more indicated by them it went to oral communication. About the credibility of the Phytotherapy, 87,0\% would start to use this treatment form after scientific confirmation, explaining the advantages and disadvantages of this application. It could be observed that the 
Phytotherapy is known among the body students of veterinary of ESAM, even so there is need of the accomplishment of new researches in this area.

Key words: Ethnoveterinary, Phytotherapy, Students.

\section{INTRODUÇÃO}

A Etnoveterinária é a ciência que envolve a opinião e o conhecimento das práticas populares utilizadas para o tratamento ou prevenção das doenças que acometem os animais (MATHIUSMUNDY E MCCORKLE, 1989). Dentre os ramos desta ciência milenar está a Fitoterapia que é o tratamento de patologias animal a base de plantas.

A Fitoterapia foi a peça essencial do arsenal terapêutico até meados do século XIX. Desde então ela foi cedendo lugar a preparados feitos com moléculas puras de elementos ativos de plantas medicinais dotadas de ação farmacológica mais específica. Assim as plantas medicinais de certo modo caíram no esquecimento e foram substituídas por terapêuticas baseadas no uso de substâncias químicas no todo ou em parte sintéticas. No entanto, os profissionais são hesitantes em integrar as práticas etnoveterinárias nos programas de criação de animais devido à falta de informações científicas válidas sobre preparação e efetividade do medicamento etnoveterinário.

O futuro profissional médico veterinário é o estudante de hoje, e é nele que a Fitoterapia deve estar presente para que seja incentivada, o primeiro a conhecer o tratamento e reconhecê-lo como válido.

Com o propósito de verificar o conhecimento dos estudantes de Medicina Veterinária da ESAM sobre a Etnoveterinária realizou-se este trabalho.

\section{MATERIAL E MÉTODOS}

O enfoque deste trabalho foi o conhecimento dos discentes de veterinária da Escola Superior de Agricultura de MossoróESAM sobre a Fitoterapia no município de Mossoró, Rio Grande do Norte, durante os meses de março a agosto de 2000.
Para o desenvolvimento deste trabalho utilizou-se a pesquisa descritiva através do método específico das ciências sociais: o método histórico, que parte do princípio que os costumes têm origem no passado sendo importante investigar os processos para verificar sua influência na sociedade de hoje.

$\mathrm{O}$ instrumento trabalhado foi à pesquisa direta através da aplicação de 138 questionários realizada na referida instituição, em todos os períodos do curso (10 períodos).

Os dados obtidos passaram por análise de frequência percentual.

\section{RESULTADOS E DISCUSSÃO}

Com esta pesquisa foi possível identificar que a Fitoterapia veterinária é do conhecimento de 73,9 \% dos estudantes de Medicina Veterinária da ESAM, sendo a faixa etária entre 15 e 24 anos a dos maiores conhecedores com 81,4 \% mostrando uma tendência a mudanças na cultura popular pois, segundo Branco e Rowlinson (1997), os mais idosos são os que têm os conhecimentos mais detalhados do uso e preparação de tratamentos tradicionais, enquanto as gerações mais jovens colocam uma maior confiança em drogas modernas ou possui um conhecimento mais limitado dos tratamentos fitoterápicos. Esta diferença possivelmente ocorreu devido a Fitoterapia está sendo mais divulgada e documentada, facilitando o acesso a informações por pessoas de menor faixa etária. Não houve variação de conhecimento com relação ao sexo, isto porque a Universidade é composta por um grupo heterogêneo e todos têm acesso as mesmas informações.

Quanto ao meio de divulgação, os estudantes afirmaram que a comunicação oral é o mais utilizado com 56,9 \%, os livros ficaram em segundo lugar com 17,6 \%; jornal e revista com 12,7 \%; a televisão participou com 11,8 \%, a internet com $1,0 \%$ e o rádio não foi citado. 


\section{REVISTA VERDE DE AGROECOLOGIA E DESENVOLVIMENTO SUSTENTÁVEL \\ GRUPO VERDE DE AGRICULTURA ALTERNATIVA (GVAA)}

Para Jirli et al. (1997), a comunicação oral feita por pais, avós, fazendeiros, amigos, etc. representa o papel mais importante na divulgação da Etnoveterinária do que a comunicação formal (revistas, jornais, livros, etc.), confirmando os resultados desta pesquisa.

Nesta pesquisa 86,2 \% dos estudantes confiam na Fitoterapia podendo passar a incentivá-la. (JIRLI et al,. 1997) tinha razão ao afirmar que a comunidade científica estava se dando conta das desvantagens e perigos dos avanços tecnológicos sem precedentes que causaram desequilíbrio ecológico e ambiental, estando dispostos a explorar alternativas para o desenvolvimento sustentável.

Apesar de 73,9 \% dos estudantes conhecerem a Fitoterapia apenas 36,2 \% usaram-na, e destes, todos obtiveram resultado eficaz, esta frequência percentual máxima de eficácia indica o valor do tratamento aplicado aos animais de Mossoró, podendo se tornar viável a prescrição destas plantas pelos futuros profissionais médicos veterinários após pesquisa mais detalhada sobre cada uma.

A planta mais utilizada (Gráfico 1) foi o mastruz (Chenopodium ambrosioides) também denominado erva de santa Maria, mastruço ou menstrus, usado como cicatrizante, antihelmíntico, antiinfeccioso, antiinflamatório, ainda no tratamento de pneumonia e traumatismos; várias dessas ações já foram comprovadas; segundo Amorim (1998), o mastruz é uma das plantas mais conceituadas na medicina popular, indicada como antiinflamatória, peitoral, estomáquica, antituberculosa. Nas contusões, é aplicada sob a forma de sumo integral ("pisada") na área afetada. O óleo essencial da erva de santa Maria encerra o ascaridol, identificado como o princípio anti-helmíntico e ao mesmo tempo tóxico, razão pela qual, extratos da planta devem ser administrados com o devido cuidado, sob a pena de provocar eventos letais. Neumann (2000) em experimentos com administração da planta a porcos constatou o desenvolvimento de lesões hepáticas, glomerulares, e tumores no estômago. $\mathrm{O}$ ascaridol provoca irritação na pele e mucosas, vômito, vertigem, dor de cabeça, danos nos rins e fígado, colapso circulatório e eventualmente morte, este óleo é um vermífugo de largo espectro, extensamente usado na medicina veterinária, porém Amorim (1998) diz que embora esta planta seja das mais conceituadas na medicina caseira no combate a verminose, a baixa eficácia demonstrada nos testes anti-helmínticos sobre $\mathrm{L}_{1}$ e $\mathrm{L}_{3}$ de estrongilídeos intestinais de equino sugere que este vegetal não oferece perspectivas promissoras para um possível controle de helmintoses de animais domésticos, na forma de extratos aquosos.

Com estas citações observa-se que há ainda muito a ser estudado sobre o mastruz, sobretudo a forma de uso, pois os efeitos colaterais estão presentes nesta planta. 


\section{REVISTA VERDE DE AGROECOLOGIA E DESENVOLVIMENTO SUSTENTÁVEL GRUPO VERDE DE AGRICULTURA ALTERNATIVA (GVAA)}

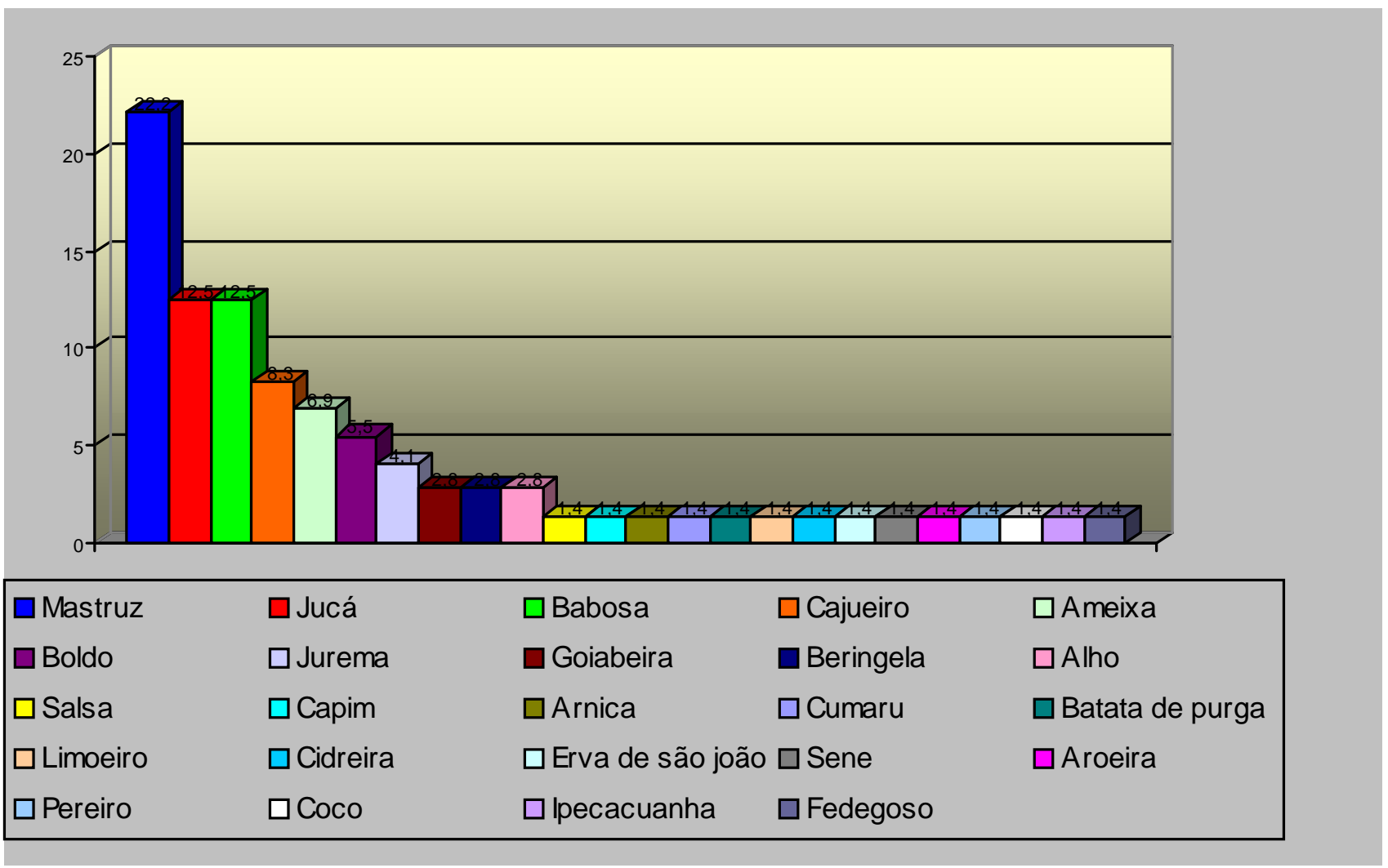

GRÁFICO 1- Demonstrativo das indicações de plantas medicinais utilizadas em animais pelos estudantes de veterinária da ESAM.

Os distúrbios da cicatrização (Gráfico 2) foi a patologia mais tratada com plantas diversas como cajueiro (Anacardium occidentalis), batata de purga (Operculina macrocarpa), entre outras.

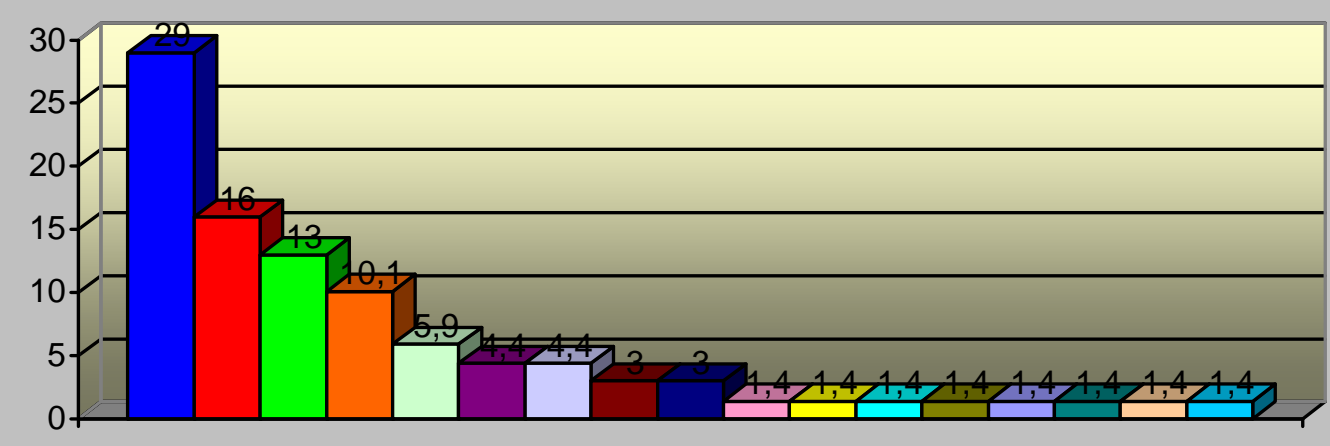

* A sarna é uma ectoparasitose porém, está separada pelo tratamento indicado ser específico para esta patologia.

\begin{tabular}{lll}
\hline Distúrbios da cicatrização & $\square$ Inflamação & $\square$ Traumatismo \\
$\square$ Endoparasitoses & $\square$ Cólica & $\square$ Diarréia \\
$\square$ Pneumonia & $\square$ Retenção de placenta & Intoxicações \\
$\square$ Infecções & $\square$ Dor & $\square$ Sarna* \\
Anorexia & $\square$ Êmese & $\square$ Ectoparasitos \\
$\square$ Miíase & $\square$ Desidratação &
\end{tabular}

GRÁFICO 2- Relação das doenças tratadas fitoterapicamente pelos estudantes de veterinária da ESAM. 


\section{REVISTA VERDE DE AGROECOLOGIA E DESENVOLVIMENTO SUSTENTÁVEL \\ GRUPO VERDE DE AGRICULTURA ALTERNATIVA (GVAA)}

As vantagens em utilizar plantas medicinais na opinião dos futuros veterinários estão demonstradas no gráfico 3; sendo o baixo custo (26,1 \%) e o menor efeito colateral (23,9\%) associado ao fato do medicamento ser um produto natural (23,9 \%), as mais citadas, revelando a preocupação deles com o animal sem esquecer do custo. Esse menor efeito colateral ainda não está bem explicado, para
Petkov (1979), tendo em vista que as substâncias biologicamente ativas das plantas são produtos do metabolismo de organismos vivos, o corpo humano consegue assimilar muitos deles com mais facilidade do que assimila remédios sintéticos estranhos. É por isso a Fitoterapia é menos prejudicial e causa menos efeitos colaterais do que as drogas químicas.

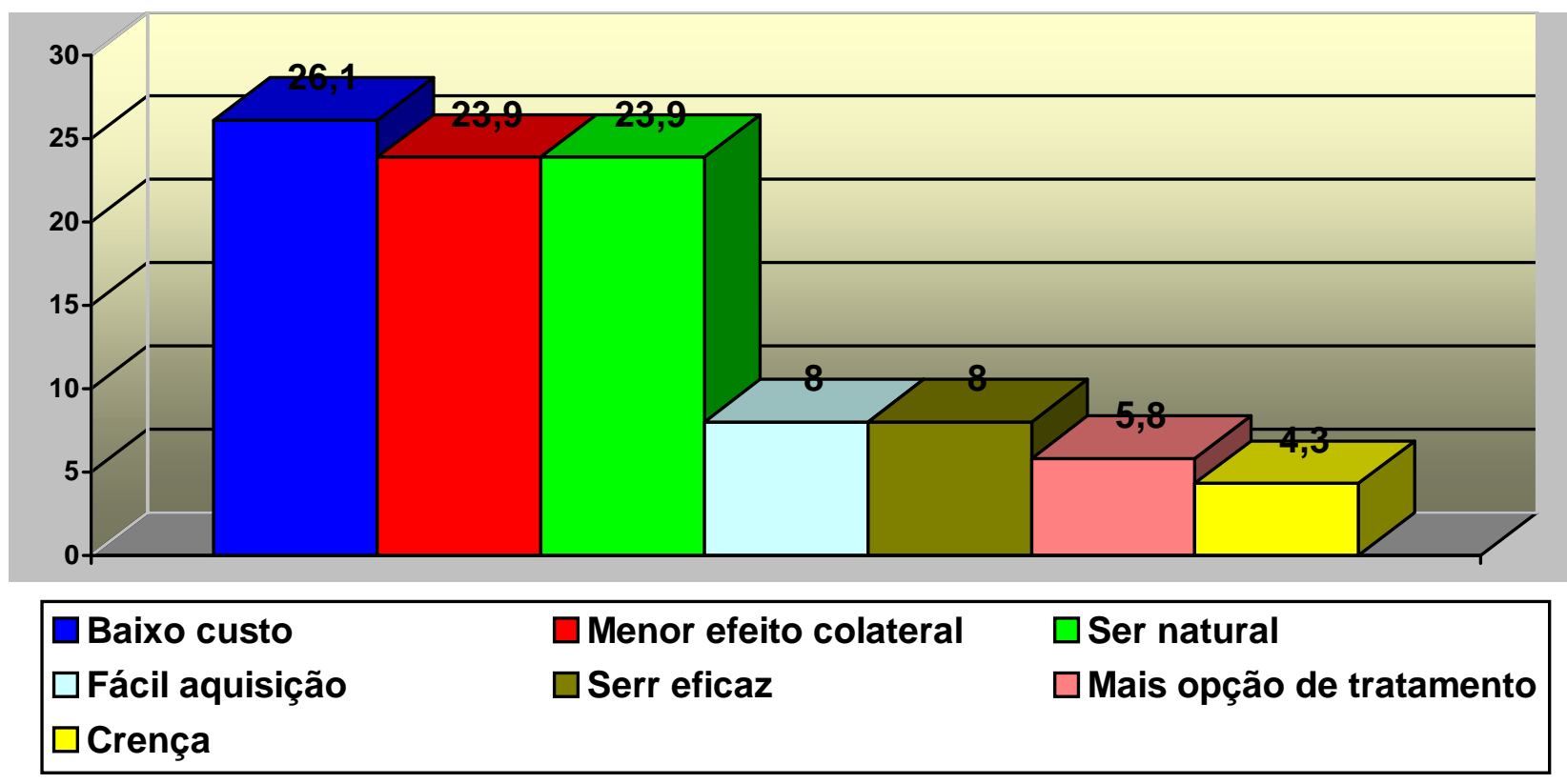

GRÁFICO 3- Demonstrativo das vantagens da Fitoterapia.

Apesar das desvantagens mostradas no gráfico 4; 23,0 \% dos entrevistados acreditam que a Fitoterapia não possui qualquer desvantagem porém, as poucas pesquisas desenvolvidas nesta área, dificultam o uso do tratamento. Em 1997, Bizimana (1997) já conhecia este problema dizendo que em contraste com o medicamento tradicional para humanos, o medicamento tradicional de uso veterinário não foi promovido ao nível internacional e poucas pesquisas foram desenvolvidas neste campo. 


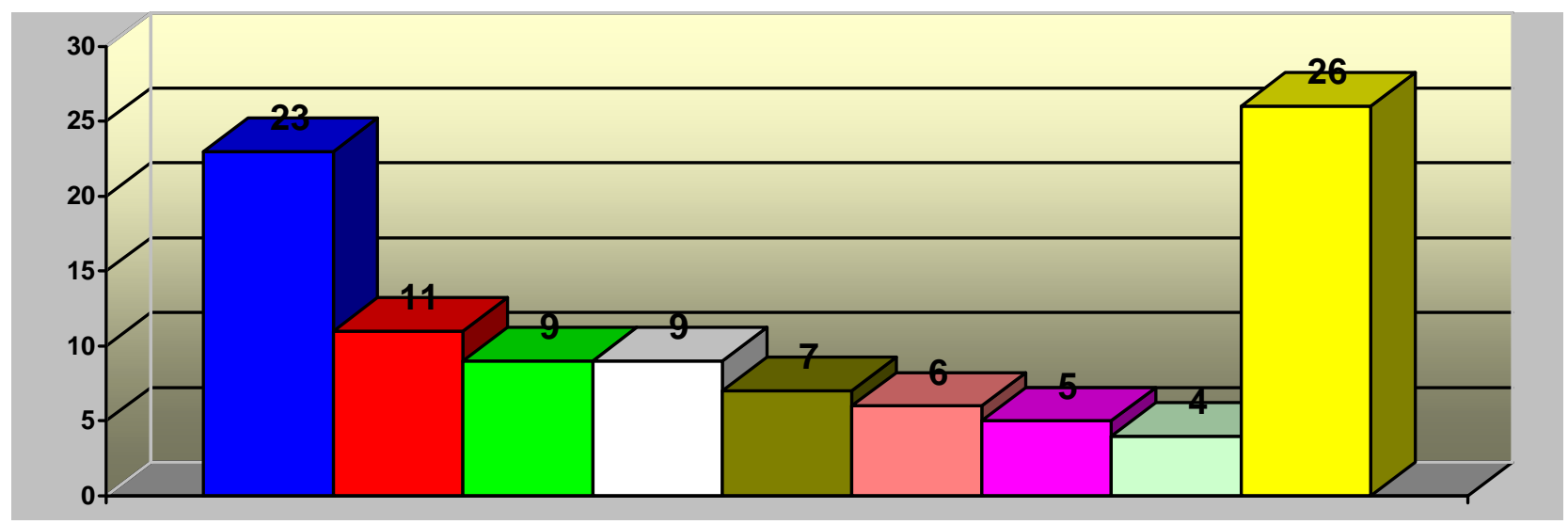

\section{Nenhuma \\ $\square$ Desconhecimento \\ $\square$ Uso indiscriminado

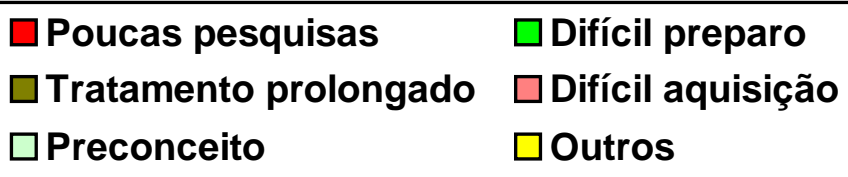 \\ Poucas pesquisas 口Outros}

GRÁFICO 4- Demonstrativo das desvantagens da Fitoterapia.

No item “Outros” estão, conjuntamente relacionadas, as desvantagens citadas uma única vez pelos estudantes, como o risco de extinção e a falta de oportunidade em utilizar esta forma de tratamento.
Na tabela 1, estão relacionadas todas as plantas citadas pelos três grupos, com seus nomes vulgares, científicos, numeração das doenças tratadas (numeração explicada abaixo da tabela) e modo de preparação. 
REVISTA VERDE DE AGROECOLOGIA E DESENVOLVIMENTO SUSTENTÁVEL

GRUPO VERDE DE AGRICULTURA ALTERNATIVA (GVAA)

TABELA 1- Relação das plantas citadas pelos entrevistados, com nome vulgar e científico, seu uso e modo de preparação.

\begin{tabular}{|c|c|c|c|}
\hline Nome Vulgar & Nome Científico & Doenças Tratadas & Modo de Preparação \\
\hline Alho & Allium sativum & 9,17 & Natural $^{4}$ \\
\hline Ameixa & Ximenia americana & $5,16,17$ & Maceração $^{5}(5,16,17) /$ sumo total $^{6}(5)$ \\
\hline Arnica & Arnica montana & $6,12,10,13$ & Decocção $^{7}$ \\
\hline Aroeira & Astronium urundeuva & 5,16 & Maceração \\
\hline Babosa & Aloe vera & $5,10,11,14$ & Sumo total $(5,20,23) /$ decocção(14) \\
\hline Batata de purga & Operculina macrocarpa & $1,5,9$ & Decocção \\
\hline Berinjela & Solanum melongena & 13 & Decocção \\
\hline Boldo & Peumus boldus & $2,8,14$ & Maceração \\
\hline Cajueiro & Anacardium occidentalis & $4,5,9$ & Decocção $(4) /$ maceração $(5,9)$ \\
\hline Capim* & - & 9 & Infusão $^{8}$ \\
\hline Coco & Cocos nucifera & 3,9 & Natural \\
\hline Cumaru & Amburana cearensis & $1,4,9$ & Maceração \\
\hline Erva de são joão & Ageratum conyzoides & 13 & Decoç̧ão \\
\hline Erva-cidreira & Lippia alba & 4 & Decocção \\
\hline Fedegoso & Cassia occidentalis & 15 & Decocção \\
\hline Goiabeira & Psidium guayava & 4 & Decocção \\
\hline Ipecacuanha & Cephaelis ipecacuanha & 9,13 & Decocção \\
\hline Jucá & Caesalpinia férrea & 5,13 & Maceração \\
\hline Jurema-preta & Mimosa hostilis & 5,13 & Maceração \\
\hline Limoeiro & Citrus limonum & 9,17 & Natural \\
\hline Mastruz & Chenopodium ambrosiodes & $5,9,10,12,13,17$ & Maceração(5,9,12,13,17)/sumo total $(23)$ \\
\hline Pereiro & Aspidosperma pirifolium & 7,24 & Sumo total (7)/maceração (24) \\
\hline Salsa & Ipomoea asarifolia & 11 & Sumo total \\
\hline Sene & Cassia angustifólia & 13 & Maceração \\
\hline
\end{tabular}

* Existem vários tipos de capim, como este não foi especificado (devido os dados serem colhidos por questionários) não se pôde classificá-lo.

${ }^{4}$ Utilizar a planta na forma in natura. ${ }^{5}$ Planta de molho em água fria por 10 a 24 horas e depois administrada. ${ }^{6}$ Folha pisada e posta sobre o local desejado. ${ }^{7}$

Colocar a planta em água fria e levar a ferver. ${ }^{8}$ Junta-se água fervente sobre a planta, cobre-se e deixa em repouso por 5 a 10 minutos.
1. Anorexia
7. Ectoparasitos
13. Inflamações
2. Cólica
8. Êmese
14. Intoxicações
3. Desidratação
9. Endoparasitos
Retenção de placenta
4. Diarréia
10. Traumatismo
11. Sarna
16. Mí́ase

6. Dor

12. Infecções 


\section{REVISTA VERDE DE AGROECOLOGIA E DESENVOLVIMENTO SUSTENTÁVEL \\ GRUPO VERDE DE AGRICULTURA ALTERNATIVA (GVAA)}

\section{CONCLUSÕES}

A Fitoterapia veterinária é do conhecimento de 73,9 \% dos estudantes de Medicina Veterináira da ESAM, porém, apenas 36,2 \% usaram este tratamento;

A comunicação oral representa o meio de divulgação mais usado para aquisição dos conhecimentos fitoterápicos;

Foram utilizadas 24 plantas no tratamento de 17 patologias, sendo o mastruz (Chenopodium ambrosioides) a mais indicada como medicinal;

O baixo custo associado ao menor efeito colateral da flora medicinal foram as vantagens de maior importância;

A Fitoterapia, não apresentou qualquer desvantagem, na opinião de 23,0 \% dos entrevistados porém, necessita-se de pesquisas nesta área.

\section{Referências Bibliográficas}

AMORIM, A. et al. Ação anti-helmíntica de plantas, XIII. Ação de extratos aquosos de Chenopodium ambrosioides L. "in vitro" sobre larvas de primeiro e terceiro estádios de estrongilídeos de equino. R. Bras. Med. Vet. Rio de Janeiro: v.20, n.1, p. 14-16. Jan/fev. 1998.

BIZIMANA, N. Scientific evidence of efficacy of medicinal plants for animal treatment. In: INTERNATIONAL CONFERENCE HELD IN PUNE. Proceedings... India: v. 2,
Ethnoveterinary medicine: alternatives for livestock development, 4-6 de November, 1997.

BRANCO, F. N.; ROWLINSON P. A.G. Use of both modern veterinary and tradicional treatments by Samburu pastoralists in northern Kenya. In: INTERNATIONAL CONFERENCE HELD IN PUNE. Proceedings... India: v. 2, Ethnoveterinary medicine: alternatives for livestock development, 4-6 de November, 1997.

JIRLI, B. et al. Cognitive domain and acceptance of ethnoveterinary medicina by animal scientists. In: INTERNATIONAL CONFERENCE HELD IN PUNE. Proceedings... India: v. 2, Ethnoveterinary medicine: alternatives for livestock development, 4-6 de November, 1997.

MATHIUS-MUNDY, E., McCORKLE, C. M. Ethnoveterinary medicine: NA annotated bibliography. Bibliography in Technology and Social Change Ames: Iowa State University, Technology and Social Change Program, n.6. 1989.

NEUMANN, A. Plantas medicinais. Disponível em: <www.jamart.com.br/plantas>. Acesso em: 21 de agosto de 2000.

PETKOV, V. Bulgária: a tradição vence o tempo. O correio da UNESCO. Rio de Janeiro: 7A, n.9, p. 39-41. Setembro/1979 